\title{
Research on Distributed Wireless Sensor Network Communication Protocol
}

\author{
Yan Zhang, Dong Xie, Guangjun Guo, Tong Jiang \\ Information School, Hunan University of Humanities, Science and Technology, Loudi, 417000, \\ China
}

Keywords: Distributed Wireless Sensor, Network, Communication, Protocol

\begin{abstract}
With the rapid development of communication technology, embedded computing technology and sensor technology and increasingly sophisticated, with perception, computing power and communication capabilities of micro-sensors began to appear in the world. By a distributed wireless sensor networks composed of these miniature sensors it aroused great concern. Such large-scale, often contain tens of thousands of nodes, and can be long-term sensor network system in unattended mode of operation with a wide range of applications in the military, environment, medical, family and business, and other fields.
\end{abstract}

\section{Introduction}

With the rapid development of communication technology, embedded computing technology and sensor technology and increasingly sophisticated, with perception, computing power and communication capabilities of micro-sensors began to appear in the world. Distributed wireless sensor networks composed from these miniature sensors (DSN, distributed sensor network) has aroused great concern. Such large-scale, often contain tens of thousands of nodes, and can be long-term sensor network system in unattended mode of operation with a wide range of applications in the military, environment, medical, family and business, and other fields [1].

Currently, the US government and foreign scientific research institutions invested heavily in the establishment launched a number of research projects on the DSN. Distributed wireless sensor networks is a concern on the current international, multidisciplinary highly cross-frontier emerging hot research field, is the perception of information collected, a revolution in process. Advances in sensor networks Based on the proposed architecture of the DSN, and focuses on a variety of challenging issues facing a new generation of sensor network communication, a more comprehensive and systematic summarized existing research results, providing further in-depth study necessary infrastructure.

\section{The Concepts and Features of DSN}

DSN is deployed by a large number of random action in the area of small sensor nodes distributed intelligent network system consisting of self-organization. Node spacing is very short, the general multi-hop (multi-hop), peer (peer to peer) wireless communication. DSN can be run in a standalone environment, it can also be connected via a gateway to the Internet, allowing users to remotely access.

DSN with traditional wireless ad-hoc (ad-hoc) network, compared with some of the following characteristics: (1) large-scale network. DSN contains thousands of nodes, several orders of magnitude than the Ad hoc network number. Very high density (2) node. Energy (3) nodes, computing power and storage capacity severely limited. (4) easily damaged nodes, the network topology changes frequently. Room (5) the use of multi-node broadcast communication, rather than point to point communications. (6) Since the number of reasons, there is no uniform node identity.

DSN the above characteristics, to design communication protocols proposed the following new problems: (1) limited resources (resource constrain). In the DSN, each node only carry a limited, non-replaceable power supply and computing, storage capacity is weak. Need for energy efficiency as the main target, the design simple protocol algorithm. (2) expandable (scalable). DSN system 
large scale, requires the communication protocol is highly scalable, able to dynamically adapt to changes in the size of the nodes, ensure the communication quality. (3) environmental adaptability (adaptability) and robustness (robustness). DSN system normally operates in unattended conditions. Changes in the environment, and energy restriction prevents vandalism error rate of the system is high. It requires communication protocol has a strong fault tolerance to keep the system stable. (4) safety (security). Safety is available to the system under the premise of the need to ensure security of communications under the premise of reducing system overhead, energy security algorithm research. (5) real-time (real-time). DSN is a reactive system, commonly used in aerospace, military, medical, etc. with strong real-time requirements, require a system with real-time communication capabilities.

\section{The Advantages and Challenges of DSN}

Advantages and challenges facing DWSN was mainly in the following five aspects.

Bad network environment and even dangerous most remote node is running normally inaccessible in the human, the sensor network nodes and to monitor the number of regions were variable, with some nodes having mobility can be monitored to the monitoring range extending monitoring area to any location of the region, and each network can overlap, effectively improves network performance monitoring, but the positioning of the sensor nodes need to be well studied.

The distance between neighboring nodes is very short to avoid long-distance wireless communication susceptible to external noise disturbances; miniaturized sensor nodes covert. A large number of sensors, although widely distributed range of features to enhance the perceived ability of the network to improve the accuracy of perception, but the sensor network hardware and software robustness and fault tolerance of a higher requirement. Especially algorithms adapt DwSN research is still lacking.

The sensor network is extremely limited supply of energy, energy can not be replaced and the sensor to transmit information to perform calculations more than energy consumption, maximize the network life cycle and sustainability is a key issue facing.

Sensor networks with self-organizing capabilities and interactive communication function, the reconfiguration and self-adjustment of the sensor is capable of high-quality requirements to complete perceptual information processing and transmission, to achieve high-quality communications capabilities; but because of the embedded processor and limited memory capacity and ability, how to effectively collaborate between nodes, sensor information processing and database queries, network broadcasting organization and management must be addressed.

The transmission mode wireless sensor networks, particularly those based IEEE1451.2, IEEE1451.5 proposed standard, that is, wireless communication protocols and TEDS (transdu.erelee-tronicdatasheet) format to build open standards for wireless sensor interface support, the sensor network focus on content.

\section{The Architecture of DSN}

According to the characteristics of DSN, comprehensive literature on the results of structural studies DSN system, this paper presents DSN architecture. The network architecture includes: composing the communication protocol modules, management modules and application support service modules of three parts. It will not only play a role in research content into a unified framework and under the system, but also to articulate the main content and composition logic sensor network research. 


\section{The Research Advances in Communication Agreement of DSN}

Physical Layer Protocol. Physical layer protocol research involves the transmission medium, select band and modulation scheme DSN uses. At present, the transmission medium DSN uses are: radio, infrared, light and so on.

Radio transmission. Radio transmission is the main way of transmission of the current DSN. Problems to be solved are: band selection, energy-efficient coding, modulation algorithm design. Frequency selection, ISM band due without having registered, with a wide range of selectable frequencies, the advantage is no specific standard can be used flexibly, had been widely used. Document gives wireless transceiver design method based on $433 \mathrm{MHz}$ and $915 \mathrm{MHz}$ two bands. In terms of coding, modulation algorithm to Binary and M-ary modulation schemes were compared. It describes the system startup time (warm up time) on the modulation energy consumption. [13] proposed based on Direct Sequence Spread Spectrum - Code Division Multiple Access (DS-CDMA) minimum energy coding algorithm. By reducing multiple access violation, reduce energy consumption.

Infrared light transmission. Compared with the radio transmission, infrared, light transmission with no complex modulation, demodulation mechanism; a receiver circuit is simple; the unit of data transmission power is small. However, due to non-transparent objects can not penetrate, can only be used in some special DSN system. In the Smart Dust project, researchers developed micro optical sensor having communication capabilities, a volume of not more than $1 \mathrm{~mm} 3$. Literature proposes two lightwave transmission mechanism: using three rectangular mirrors (CCR) passive transmission mode and uses a laser diode, active transmission mode easy to control mirror. The former need to install a light source, configure CCR to complete the communication. The latter uses a laser diode and the master laser communication systems to transmit data. Currently, the study of DSN physical layer protocol is still in the initial stage, the urgent need to address the problem: to reduce hardware costs need to study integrated, all-digital, universal circuit design methods; in energy efficiency need to be designed with a high data rate low symbol rate coding, modulation algorithm.

Data Link Layer Protocol. DSN topology can be divided into two types, namely: the planar structure; hierarchy. In a planar structure, all network nodes in the same equal status, there are no differences of rank and level, it is sometimes also referred to as peer to peer structure. Planar structure is relatively simple and does not require any structural maintenance work; equal status of network nodes, difficult to produce a bottleneck effect, it has good robustness. A typical planar structure generation algorithm: ASCENT, SPAN, GAF, STEM and so on. The biggest drawback is that the planar structure of the network: no network node management, self-organizing complex algorithm to work, the network dynamic response is slow. With a planar structure corresponding to the hierarchy.

Hierarchical networks are generally in the form of clusters exist, a collection of so-called cluster, is the network node associated with a certain composition. In the cluster, there is usually a certain election rules, known as cluster head node for management or control of the set of nodes; except cluster head node, the other nodes within a cluster node may be referred to the members, a hierarchy is a central structure with no center complex structure, scalability, and ease of management features. Sohrahi proposed a single cluster structure generation algorithm [18]. Single cluster structure similar to the center and is not suitable for use in large-scale DSN system. Sohrahi proposed to use two channel system is divided into two clusters of two-channel grouping mechanism (DNC) and collections grouping mechanism (RCA). Caccamo use system is divided into a plurality of clusters, the use of FDMA, adjacent clusters use different frequencies to communicate. The disadvantage multibank structure are: inter-cluster communication complexity, high cost of hardware.

Routing Protocol. Younis et al proposed routing protocol is based on three-tier architecture. The difference is that with $\mathrm{LEACH}$, the agreement requires that the network run by the end user before the sensor nodes into clusters, and notifies the location information and the identification ID assigned to each node in the cluster cluster head node. Node within the cluster may be the presence of one of the following four ways: perception, forwarding, forwarding and perception, sleep. 
Energy cluster head is not limited, it can monitor the nodes energy changes, determine and maintain the four states of the sensor, and take advantage of the cost function as a link cost, select the least cost path as the best path nodes communicate with. The simulation analysis, the protocol has good energy performance during operation, high throughput and low latency communication.

Transport Protocol. Because DSN own weaknesses in communication reliability, so that face great difficulties in achieving its transmission control. Stage of research focused on the transmission control error recovery mechanisms. Document analysis-end error recovery performance in a wireless multi-hop network. Simulation results show that, with the decline in the quality of the radio channel (channel error rate from $1 \%$ to $50 \%$ ), end to end error recovery mechanism performance drops quickly (transmission success rate dropped from $90 \%$ to close to 0 ). To this end, the paper proposes a hop (hop by hop) error recovery mechanisms. By the intermediate node involved in data transmission error detection and recovery, so that the data at each hop transmissions are checked for errors and to avoid the accumulation of errors by the communication quality degradation caused. In order to ensure there is enough lost packets retransmission time, the paper also designed the data flow control mechanism PSFQ one kind of slow cache stored. Through each intermediate node in the data path in both maintain the correct packet forwarding order to reduce the wrong sequence packets blindly forwarding. The method can effectively balance the packet buffer number of intermediate nodes, providing a low overhead for the DSN error recovery services. Current research on DSN transmission control little. How in the topology, dynamic channel quality conditions change, providing for the upper application of energy-saving, reliable, high real-time data transmission services is the focus of future research.

\section{Conclusions}

Flexible, robust, scalable and rapid deployment features such as DSN in the military, the environment, health care, family and other commercial areas have a high value. It is foreseeable, DSN will become part of the future of people's lives inseparable. Resources are limited, unattended, and so easily damaged node to DSN realization brought a lot of difficulties. This paper studies predecessors, given the architecture of the DSN, and within the framework of the architecture, described in detail the function of each communication protocol module, analysis and comparison of the existing design ideas and solutions strategies. At present, the people of DSN is still in its infancy, there are still many urgent problems to be studied further.

\section{Acknowledgements}

This work is supported by the Research Foundation of Education Committee of Hunan Province, China (15C0724), Science and Technology Planning Project of Loudi Municipal Government, Hun an Province, China (2015RW03), and the Construct Program of the Key Discipline "Computer App lication Technology" in Hunan Province, China.

\section{References}

[1] XU N: IEEE Computer Magazine, Vol. 2 (2009) No 53, p.25-26

[2] AKYILDIZ I F: Computer Networks, Vol. 12 (2010) No45, p.83-85

[3] Meng Li IEEE Computer Technology, Vol. 2 (2013) No 59, p.102-105

[4] Shuang Wu: Computer Information, Vol. 2 (2011) No 43, p.29-43

[5] Ling Liu: Computer Information Resources, Vol. 12 (2014) No 22, p.76-80 